\title{
A CLASS OF WEIGHT FUNCTIONS FOR WHICH TCHEBYCHEFF QUADRATURE IS POSSIBLE
}

\author{
BY J. L. ULLMAN
}

\author{
Communicated by R. C. Buck, June 13, 1966
}

1. Introduction. By a weight function $W(x)$ we mean a real valued nonnegative function on $[-1,1]$ for which the proper or improper Riemann integral exists and has the value one. If the equations

$$
\frac{1}{n} \sum_{i=1}^{n} x_{i, n}^{k}=\int_{-1}^{1} x^{k} W(x) d x, \quad k=1, \cdots, n,
$$

have real solutions for all positive integers $n$, we say Tchebycheff quadrature is possible for the weight function $W(x)$. A result attributed to Hermite [1] is that Tchebycheff quadrature is possible for

$$
W(x)=\frac{1}{\pi\left(1-x^{2}\right)^{1 / 2}},
$$

but the author has found no other weight function having this property in previous literature. The object of this note is to announce the explicit structure of an infinite family of Tchebycheff weight functions, and to sketch the proof. Full details will appear elsewhere.

2. TheOREM. Tchebycheff quadrature is possible for

$$
W(x)=\frac{1}{\pi\left(1-x^{2}\right)^{1 / 2}} \frac{1+2 a x}{1+4 a^{2}+4 a x},
$$

where $-1 / 4 \leqq a \leqq 1 / 4$.

Sketch of Proof. Lemma 1 describes a general method for investigating the solutions of equations (1). Lemma 2 derives a special fact that facilitates the use of Lemma 1 for the case of the weight function defined by (2). Lemma 3 combines the previous information to complete the proof.

Lemma 1. Let $W(x)$ be a weight function and let $m_{k}=\int_{-1}^{1} x^{k} W(x) d x$, $k=0,1, \ldots$. Let

$$
f(z)=z\left(-\sum_{k=1}^{\infty} \frac{m_{k}}{k z^{k}}\right) .
$$

For a positive integer $n$ let $T_{n}(z)$ be the polynomial part, including 
the constant term, of the Laurent expansion about infinity of $(f(z))^{n}$. If we write

$$
T_{n}(z)=\prod_{i=1}^{n}\left(z-z_{i, n}\right),
$$

then the numbers $z_{i, n}, i=1, \cdots, n$ satisfy the equations

$$
\frac{1}{n} \sum_{i=1}^{n} z_{i, n}^{k}=m_{k}, \quad k=1, \cdots, n .
$$

Lemma 2. Let $\left(z^{2}-1\right)^{1 / 2}$ denote the analytic function whose domain of definition is $E_{1}$, the z plane with the interval $[-1,1]$ deleted, and which is determined by requiring that the values be positive for $z$ real and greater than one. Then for $|z|>1$, and for $-1 / 2<a<1 / 2$,

$$
\frac{z+\left(z^{2}-1\right)^{1 / 2}}{2}+a=z \exp \left(-\sum_{k=1}^{\infty} \frac{m_{k}}{k z^{k}}\right),
$$

where $m_{k}=\int_{-1}^{1} x^{k} W(x) d x, k=1,2, \cdots$, and with $W(x)$ given by (2).

Lemma 3. Let $n$ be a positive integer and let $T_{n}^{a}(z)$ be the polynomial part including the constant term of the Laurent expansion about infinity of $(f(z))^{n}$, where $f(z)$ is given (3) for the choice of weight function (2). Then if $-1 / 4 \leqq a \leqq 1 / 4, T_{n}^{a}(z)$ has real zeros.

Comment on Lemma 3. In forming the polynomial part including the constant of the Laurent expansion about infinity of $(f(z))^{n}$, we can operate on

$$
\left(\frac{z+\left(z^{2}+1\right)^{1 / 2}}{2}+a\right)^{n}
$$

by Lemma 2. The Laurent expansion about infinity of this expression has the same polynomial part including constant as the Laurent expansion about infinity of

$$
\left(\frac{z+\left(z^{2}-1\right)^{1 / 2}}{2}+a\right)^{n}+\left(\frac{z-\left(z^{2}-1\right)^{1 / 2}}{2}+a\right)^{n}-a^{n},
$$

but this latter expression is a polynomial, and hence is $T_{n}^{a}(z)$. If $-1 / 4 \leqq a \leqq 1 / 4$, the substitution of $z=\cos \theta$ in (5) reveals that $T_{n}^{a}(\cos \theta)$ is zero for $n$ values of $\theta$ in $[0, \pi]$ so that $T_{n}^{a}(z)$ has real zeros. Thus the proof of the theorem is complete.

3. Concluding remarks. Because of the existence of this class of 
Tchebycheff weight functions, it now becomes of renewed interest to consider the problem of characterizing all Tchebycheff weight functions. The author intends to return to this more general problem.

\section{REFERENCE}

1. P. L. Tchebycheff, Sur les quadratures, Collected works, Vol. II, Chelsea, New York, 165-180.

UNIVERsity OF Michigan 